\title{
Adam Trybus
}

\section{A GENERALISATION OF A REFUTATION-RELATED METHOD IN PARACONSISTENT LOGICS}

\begin{abstract}
This article describes a refutation method of proving the maximality of three-valued paraconsistent logics. After outlining the philosophical background related to paraconsistent logics and the refutation approach to modern logic, we briefly describe how these two areas meet in the case of maximal paraconsistent logics. We focus on a method of proving maximality introduced in [34] and [37] that has the benefit of being simple and effective. We show how the method works on a number of examples, thus emphasising the fact that it provides a unifying approach to the search for maximal paraconsistent logics. Finally, we show how the method can be generalised to cover a wide range of paraconsistent logics. We also conduct a small experiment that confirms the theoretical results.
\end{abstract}

Keywords: paraconsistency; refutation; maximality

\section{Introduction}

Paraconsistent logics are a tool used for the formal treatment of inconsistent theories outside classical logic. In them, the so-called explosion law, which holds true in classical logic, is rejected. Arising from the critique of how classical formal logic deals with contradictions, paraconsistent logics form a wide array of systems. Paraconsistent logics that retain as many classical laws as possible have proved to be of special interest. The maximal paraconsistent logics, as they are known, can also be adequately described in terms of what they reject from classical logic, rather than what they retain. This allows one to use the methods from the refutation calculus in order to analyse such systems. Refutation focuses on 
non-validity and thus often proves to be a useful, if somehow neglected, formal approach. In the first part we briefly describe the development of paraconsistent logics with the emphasis on the maximal ones. We then move on to outline the main ideas behind the refutation calculus. We end this part with describing the research on maximal subclassical paraconsistent logics. The next part is a detailed description of a specific method, utilised in the refutation calculus that can be used to easily prove the maximality of some three-valued paraconsistent logics. Our main contribution lies in showing how this method generalises, allowing one to prove the maximality of a vast number of such logics, thus providing a simple and unifying account. Finally, we round up with a description of a computational analysis of chosen three-valued matrices that confirms the theoretical results obtained here.

\section{Background}

\subsection{Paraconsistency}

The idea that logic is based on a number of non-demonstrable, fundamental laws (principles) can be traced back at least to Aristotle. It has been argued that such laws were originally connected to certain assumptions on the relations between entities and their mode of existence (cf. [7, p. 10-14]). Aristotle considered the so-called law of contradiction to be one of the most important laws of thought and being (cf. [18, p. 3]). In terms of classical propositional logic, this law can be expressed as $\neg(A \wedge \neg A)$, where $A$ is any well-formed formula. Aristotle's ideas became the received wisdom in terms of how logic was viewed and developed within the European tradition in the centuries to come. Things began to change drastically, however, with the dawn of the previous century. Jan Eukasiewicz, one of the most famous members of the Lvov-Warsaw school of logic, took a renewed interest in the law of contradiction and famously stated that a third period in the history of this law is approaching, where it is to be revised in a manner similar to the revision of the parallel postulate in geometry (cf. $[18$, p. 7$]$ ). This is very similar to what Nicolai Vasil'iev had stated some years before:

Euclidean geometry is the geometry of our world [...] but there could be other, imaginary worlds, whose geometry is non-Euclidean. Similarly, Aristotelian logic is the logic of our world but there could be imaginary worlds, whose logic is imaginary logic.

[25, p. 139] 
For that reason, Vasil'iev is now considered a forefather of non-classical logic. It should be noted however that his ideas were not as influential as those of Eukasiewicz. Vasil'iev worked in the framework of Aristotelian syllogistic and focused on extending it. He introduced a new kind of proposition, which he called accidental and connected with it a new category of quality (on top of the traditional affirmative and negative) indifference. In this view, there can be imaginary worlds, where many of the traditional laws of logic can be non-valid; however there is a core of laws that have to be valid in all worlds. This includes the law that forbids an agent to contradict herself/himself. Yet, the law of contradiction is compromised as the indifferent propositions are true only if $S$ is $P$ and non- $P$ at the same time (where $S, P$ are standard names for terms in Aristotelian syllogistic). ${ }^{1}$ Also, in imaginary logic, the so-called law of excluded middle (everything is $P$ or non- $P$ ) is replaced by the law of excluded fourth: everything is $P$, or non- $P$, or $P$ and non- $P$ (cf. [25, p. 138]). It can be argued that Vasil'iev and Łukasiewicz were the first to explicitly take issue with the law of contradiction but there are a number of thinkers who, over the years, had had implicitly questioned the law, starting with Heraclitus, Antisthenes the Cynic, Nicholas of Cusa all the way to G. W. F. Hegel. This obviously assumes the Eurocentric point of view, where philosophy is viewed as more or less continuous development of ideas radiating from Ancient Greece, which is severely limiting at best and harmful and dishonest at worst. More recently, there has been some research righting this wrong, investigating the non-European approaches to what we call the law of contradiction. Most efforts have focused on the analysis of the so-called Jaina logic (cf. [12, p. 267]). Jaina was one of the several philosophical schools in classical India more or less intensively developed for about a thousand years starting from the second century before the common era. Jainas believed that philosophical discussion is a collection of various views (standpoints), some of which can contradict each other. Their approach is often called pluralistic:

The Jaina philosophers support their pluralism by constructing a logic, in which there are seven semantic predicates [...]. The seven-predicate theory consists in the use of seven claims about sentences, each preceded by "arguably" or "conditionally".

$[12$, p. 269]

1 There are also alternative interpretations of Vasil'iev's approach, according to which the law of contradiction is not tampered with in his system (see [38, p. 134]). 
In the seven-valued Jaina logic, there are three basic truth values (truth, false, non-assertible) with the rest being a combination of the basic ones. The discussion on how to fit this system in terms of the more modern developments in logic is still ongoing (cf. [12, p. 271-272]).

It goes without saying that the non-European approaches fell outside the purview of Łukasiewicz. Speaking about his European predecessors, he complained that the worst thing was that this law had never been truly justified (cf. [18, p. 6]). As a result of his investigations, he distinguished three forms of the law of contradiction: (i) ontological (no object can have and not have the same feature at the same time), (ii) logical (two statements, one asserting that a given object has the feature and the other asserting that it has not, cannot be both true), and (iii) psychological (two beliefs, corresponding to the contradictory statements, cannot exist at the same time in one mind) (cf. [18, p. 9 14]). He focuses on the first two forms (stating that the first is more fundamental) and waves the third one off as at most an empirical law (cf. [18, p. XXIV]). Eukasiewicz also showed that the law of contradiction, in its logical form, is not, as stated by Aristotle and repeated many times by his followers, a basic, self-evident law of logic: it is, in fact, based on a number of other statements. Łukasiewicz's approach has been the subject of re-interpretation and critique itself (cf. [21, 22]), yet his impact on the development of logic in that respect in undeniable. Other thinkers, of a more philosophical persuasion, also joined ranks in critiquing the received wisdom, For example, Ludwig Wittgenstein, one of the most important philosophers of the twentieth century, expressed views in favour of alternative approaches to logic in the following manner:

Indeed, even at this stage, I predict a time when there will be mathematical investigations of calculi containing contradictions, and people will actually be proud of having emancipated themselves from consistency.

(quoted after [24, p. 223])

Thus, Wittgenstein, in this fleeting remark, goes even further than Łukasiewicz in terms of embracing the inconsistent. This all shows that the early twentieth century was a period where alternative ways of thinking about what logic is began to emerge on the outskirts of what became known as classical formal logic (as initiated by Russell and Whitehead in their Principia). It brewed for couple of decades and took the shape of various formal systems as part of the expanding field of non-classical logic. However, it began to take a different approach to the problem at 
hand, coming from a more direct analysis of the world around it. For example, researchers claimed that inconsistencies have to be treated as inherent parts of many theories (including scientific ones). One can divide the more recent motivations for developing alternative systems into three groups: (1) ontological - we do not have any sound reasons for believing that the world we live in is internally consistent (on the contrary, our every-day experiences as well as more advanced scientific theories, e.g. quantum mechanics, seem to confirm that the structure of the world is indeed inconsistent); (2) epistemological - there are many situations, when we can assume that information we obtain is incomplete and hence potentially inconsistent but where we still want to, or sometimes have to, reason using the information at our disposal, even in the face of contradiction; and (3) methodological - even a superficial analysis of the history of mathematics and the development of natural sciences shows that searching for contradictions (and getting rid of, or perhaps just containing, them) is one of the most distinctive features of the process of coming up with a sound theory. It has been also raised that the classical logic cannot properly handle contradictory theories. This is because of another classical law, stating that ex contradictione (sequitur) quodlibet, or that from contradiction everything follows. It is often abbreviated to $E C Q$ and takes shape of the following formula: $A \rightarrow(\neg A \rightarrow B)$, where $A$ and $B$ are any well-formed formulas. In modern parlance, this means that the set of consequences of an inconsistent theory is infinite and contains all the well-formed formulas. For that reason, this law is sometimes known as the explosion law, since the inconsistent theory can be thought of as exploding with consequences, hence becoming useless on the grounds of classical logic. This led some to claim that:

$[\ldots]$ classical logic is on the wrong track as far as living with our best theories is concerned, and that it is equally on the wrong track when it comes to removing inconsistencies from our knowledge.

[5, p. 12]

This proved to be a motivation for many researchers that tried to come up with the systems of logic allowing us to deal with inconsistent theories and prevent them from exploding with consequences. Such systems became known as paraconsistent and are experiencing a period of rapid development. $^{2}$ Proponents of various paraconsistent logics emphasise different motivations behind their systems (as mentioned above); some

${ }^{2}$ With the benefit of hindsight, several systems developed in the past can also be claimed to be paraconsistent. We should note here the three-valued logic developed by 
even finding their way into more practical applications in computer science or the foundations of mathematics. In terms of philosophy, this led to the emergence of various schools of paraconsistent logic with the main centres in Poland, Brazil, Belgium, and Australia (cf. [4]). The Polish (or modal) school is related to the work of Stanisław Jaśkowski and his discussive logic (see, e.g., [17]). The Brazilian school is connected with the name of Newton C.A. da Costa, who proposed what he calls the principle of tolerance in mathematics:

From the syntactical and semantic points of view, every theory is permissible, since it is not trivial. Broadly speaking there exists in mathematics what is not trivial. (quoted after [4, p. 105])

One of the distinctive features of the Belgian approach is its practical bent. The solutions proposed by the Flemish logicians stem from methodological, rather than ontological, convictions. The main motivation is that we cannot be sure whether the theories of the present would not turn out to be inconsistent in the future but we need a way of working with them as these are the best tools currently at our disposal. Hence the need to control inconsistencies and contain them (see [6]). This approach is broader than the scope of paraconsistent logic but the most well-known development does fall within the so-called inconsistency-adaptive logics. The Australian approach, on the other hand, is strongly related to a philosophical standpoint called dialetheism, stating that true contradictions really exist (the book [23] lays the foundations of this approach; see also [27]). There are, obviously, many other places, where research on paraconsistency takes place these days, making it a truly world-wide endeavor (cf., e.g., $[21,21,39,4]$ ). What is common to all those developments from a formal point of view is that the law of explosion is rejected. We emphasise this as it connects to the other thread we wish to elaborate on, namely the notion of rejection, or refutation, in logic.

\subsection{Refutation}

The notion of rejection in logic can also be traced back to the works of Aristotle, meaning it has been with logic from the very beginning. In contrast to the debate on the status of the law of contradiction however, this part of Aristotle's considerations has not been the subject of any

Eukasiewicz, however with different motivations in mind, but also systems proposed by I. E. Orlov (cf. [1, p. 19]) and A. N. Kolmogorov. 
substantial analysis over the ages. In fact, it had remained almost unnoticed until the first half of the twentieth century. It was - again - Jan Łukasiewicz, who teased out the details and developed further the ideas of Aristotle.

Of two intellectual acts, to assert a proposition and to reject it, ${ }^{3}$ only the first has been taken into account in modern formal logic. Gottlob Frege introduced into logic the idea of assertion, and the sign of assertion $(\vdash)$, accepted afterwards by the authors of Principia Mathematica. The idea of rejection, however, so far as I know, has been neglected up to the present day.

$[19$, p. 94$]$

Eukasiewicz observed that Aristotle's approach relates not only to asserting the valid syllogisms but also to rejecting the non-valid ones. Eukasiewicz attempted to formulate refutation rules for Aristotle's syllogistics and treat the constructed system as a dual to the standard one. Łukasiewicz's work took into account the tools of propositional logic. In the process, two rules were introduced: reverse modus ponens (if $A \rightarrow B$ is asserted and $B$ rejected, then so is $A$ ) and reverse substitution (if $A$ is a substitution of $B$ and $A$ is rejected, then so is $B$ ) which, together with a single rejection axiom $p$ formed the first formal refutation calculus for the classical propositional logic $[19$, p. 71$]{ }^{4}$ The idea of rejection is also present in some earlier works by this author. For example, in [20] he introduces both: a symbol for assertion and a symbol for rejection (stating that he is following Brentano in that, cf. [20, p. 89]); however he is not developing a rejection system there and merely uses the rejection symbol to single out non-theorems (cf. [20, p. 100, 104]). We note that Łukasiewicz also worked on several non-classical logics in that context, including intuitionistic and modal systems (see [41, p. 191-192]). Later on, another Polish logician, J. Słupecki, showed that the rules proposed by Eukasiewicz for the Aristotelian syllogistics are not sufficient to reject all the invalid syllogisms and proposed his own rule set (cf. [41]). It should be said that more contemporary research on refutation focuses on propositional logic, mostly in the context of Hilbert-style systems. Słupecki's work spanned various logical systems and he established a small

3 Łukasiewicz credits Brentano for this distinction.

${ }^{4}$ We should add that the notion of rejection is also made use of by Carnap. He states: "One new syntactical concept which might be added to those used in customary calculi is $C$-false. It is defined on the basis of 'directly $C$-false' which is defined by rules of refutation" [9, p. 101]. 
research centre dealing with refutation-related problems. More recently, refutation techniques have been a subject of a series of publications dealing with a wide range of non-classical logics (see also the next section), ranging from intuitionistic and related systems (cf. [30, 31, 33]) to numerous modal systems (cf., e.g., $[32,36]) .{ }^{5}$ Apart from that, however, the work on refutation systems is sporadic. The paper [8] presents an alternative rejection system for the classical propositional logic, whereas the papers $[13,14]$ deal with rejection in modal logics. One should also note an attempt at using the notion of rejection in the framework of argumentation networks (cf., e.g., [11]).

\subsection{Refutation and paraconsistency}

As far as we know, the problem of rejection in paraconsistent logic is not a subject of any long-term research programme. The publications [34] and [37] apart from constructing refutation systems for various paraconsistent logics also point to one of the areas, where both research on paraconsistency and refutation meet in a natural way. To discuss this, we need to set up the scene first. As it has been mentioned, one is faced with a plethora of paraconsistent logics, developed with various motivations in mind. It is unavoidable, however, that some such systems receive more attention than others. Indeed, this is especially true of certain subclassical paraconsistent logics. Roughly speaking, a paraconsistent logic is subclassical if either the set of all its valid formulas or its consequence relation is a proper subset of the set of theorems or the consequence relation of classical propositional logic, respectively. Among the subclassical logics, the maximal ones hold a special place. In broad terms, a paraconsistent subclassical logic is maximal if there is no other, different, paraconsistent subclassical logic with a set of theorems or a consequence relation properly containing its set of theorems or its consequence relation, respectively.

Refutation considered in a narrow sense is tied to building Hilbertstyle systems of rejection axioms and refutation rules and analysing the properties of such systems. However, in this article we employ a broader sense of the term, where refutation is considered as providing a certain lens through which to view logical problems. The applicability of this broader view is brought to the fore in the case of maximality: there is

${ }^{5}$ Cf. the chapter [35], which contains a detailed formal description of various propositional refutation systems. 
a simple and elegant way of expressing maximality in terms of rejecting what we do not want, rather than focusing on the positive criteria. Informally, maximal logics are those that reject those formulas valid in classical logic that are, in one way or another, tied to ECQ or the nonparaconsistent rule of inference (details follow in section describing the results on the maximality of theory). The papers [34] and [37] use the techniques developed for proofs related to refutation systems to show maximality of a number of paraconsistent logics. Again, this is not to say that these are refutation-specific in the sense of being used only in the context of creating refutation systems. In fact, the crucial elements of method we focus on here were used before in settings not related to refutation, as they essentially boil down to defining certain substitutions (see [40]). For a number of years, however, this method has been used with success in solving logical problems as viewed from the refutation-oriented point of view (see e.g. [35]). This article deals with paraconsistent logics, which is indeed the setting in which the method is applied in [34] and [37]. In what follows, we give examples of this method in relation to some paraconsistent logics not analysed in the above and show how more general results can be obtained with the use of the method, establishing the maximality of a greater number of paraconsistent logics in one fell swoop. It should be made clear that we take the matrix approach to defining logics as this seems the most conducive to what we are trying to achieve (and is, after all, the usual practice). However, this also introduces an important constraint: maximality as described by us can potentially be a feature of certain paraconsistent logics that cannot be defined using the matrix approach. Therefore, we lay no claim to capturing maximality in all its complexity but instead, by working within the confines of our chosen method, we wish to focus on identifying certain classes of matrices that give rise to maximal logics. Finally, we note that in this presentation, for the most part we limit ourselves to the case of three-valued logics with matrix semantics defined for two primitive connectives: $\neg$ and $\rightarrow$.

\section{Results}

\subsection{Syntax and semantics}

Let $\mathcal{L}$, a set of symbols, consist of two connective symbols $\neg$ and $\rightarrow$; a countably infinite stock of propositional symbols $\mathcal{A T}$ (lower case letters, indexed if needs be); and various bracket symbols (consistently with the 
usual practice). Any finite concatenation of the elements of $\mathcal{L}$ is called an expression. An expression formed according to the following rules is called a formula. All members of $\mathcal{A T}$ are formulas (sometimes called atomic formulas). If $A$ is a formula then so is the expression $\neg A$. If $A$ and $B$ are formulas, then so is the expression $(A \rightarrow B)$. We omit the brackets according to the well-known rules that we do not state here for want of space. Taking the notion of a subformula for granted, by $\mathcal{S}_{B}$ we denote the set of all atomic subformulas of a given formula $B$.

Let $\mathfrak{M}=\left\langle M, D, \neg \mathfrak{M}, \rightarrow_{\mathfrak{M}}\right\rangle$, where $D \subseteq M, \neg \mathfrak{M}: M \longrightarrow M$ and $\rightarrow_{\mathfrak{M}}: M \times M \longrightarrow M$. We call $\mathfrak{M}$ a (logical) matrix. ${ }^{6} \quad M$ is called a domain, $D$ is the set of distinguished values and $\neg \mathfrak{M}, \rightarrow_{\mathfrak{M}}$ provide interpretations for the connective symbols in $\mathcal{L}$. We say that the matrix is $n$-valued if $|M|=n$. Throughout this article, however, whenever we talk about an $n$-valued matrix, we always mean $n>2$.

A valuation is any function $v$ of the form $v: \mathcal{A T} \longrightarrow M$, where $\mathcal{A} \mathcal{T}$ is the set of all atomic formulas. Given a valuation function $v$, it can be extended to all formulas in the following way: $v(\neg A)=\neg \mathfrak{M} v(A)$ and $v(A \rightarrow B)=v(A) \rightarrow_{\mathfrak{M}} v(B)$. Let $\mathcal{V}_{\mathfrak{M}}$ be the set of all the valuations as defined for $\mathfrak{M}$. We write $\models_{\mathfrak{M}} A$, and say that $A$ is valid (in $\mathfrak{M}$ ), when $v(A) \in D$, for all $v \in \mathcal{V}_{\mathfrak{M}}$.

Define $\mathcal{T}_{\mathfrak{M}}=\left\{A: \models_{\mathfrak{M}} A\right\}$, that is $\mathcal{T}_{\mathfrak{M}}$ is a set of all valid formulas of $\mathfrak{M}$, called the theory of $\mathfrak{M}$. Every logical matrix determines a consequence relation $\vdash_{\mathfrak{M}}$ between a set of formulas $\mathcal{X}$ and a formula $A$ in the following manner. For all $X \in \mathcal{X}$ and all $v \in \mathcal{V}_{\mathfrak{M}}$, if $v(X) \in D$, then $v(A) \in D$. In such a case we write $\mathcal{X} \vdash_{\mathfrak{M}} A$. Formula $A$ is valid if and only if $\emptyset \vdash_{\mathfrak{M}} A$. The above encapsulates the two meanings of the word logic used in this article: either as a set of valid formulas or as a consequence relation. We use this generic term whenever we are not interested in differentiating between these two senses, otherwise we talk about theory or consequence respectively. We refer to a logic (in any of the distinguished senses) as being $n$-valued, if it is defined using an $n$-valued logical matrix. For the sake of brevity, we take the liberty of referring to a logical matrix as a logic whenever it does not lead to misunderstanding.

${ }^{6}$ Perhaps it would have been more proper to refer to such arrangements as classes of matrices where the two connectives are defined in a specified way, which can then be further distinguished by extending them with the definitions of additional connectives, especially in the view of our approach presented in the section on computational analysis, but we drop this distinction as essentially insignificant. 
Let $\mathfrak{C}=\langle\{1,0\},\{1\}, \neg \mathfrak{C}, \rightarrow \mathfrak{C}\rangle$ with the connectives interpreted classically and the standardly defined valuation function. $\mathcal{T}_{\mathfrak{M}}$ is paraconsistent if $A \rightarrow(\neg A \rightarrow B) \notin \mathcal{T}_{\mathfrak{M}} ;{ }^{7}$ it is subclassical if for every formula $A$ we have $A \in \mathcal{T}_{\mathfrak{M}}$ only if $A \in \mathcal{T}_{\mathfrak{C}}$; and it is maximal if there is no other subclassical paraconsistent theory $\mathcal{T}^{\prime}$ such that $\mathcal{T}_{\mathfrak{M}} \subset \mathcal{T}^{\prime}$. $\vdash_{\mathfrak{M}}$ is paraconsistent if there are formulas $A, B$ such that $\langle\{A, \neg A\}, B\rangle \notin \vdash_{\mathfrak{M}}$; it is subclassical if $\vdash_{\mathfrak{M}} \subset \vdash_{\mathfrak{C}}$; and it is maximal if there is no matrix $\mathfrak{M}^{\prime}$, such that $\vdash_{\mathfrak{M}^{\prime}}$ is paraconsistent and $\vdash_{\mathfrak{M}} \subset \vdash_{\mathfrak{M}^{\prime}} \subset \vdash_{\mathfrak{C}}$. In this article we deal with logical matrices that are related to subclassical paraconsistent theories and consequence relations and focus on what conditions should be imposed on such matrices to lead to the maximality of both the associated theories and consequence relations.

\subsection{The method}

The method we are about to utilise, and which has been so far described only in broad terms hinges on the following theorem. It is a formalisation of the refutation-related intuitions of what it means for a logic to be maximal. Let $\mathfrak{M}$ be such that for any formulas $A, B$ and any valuation $v$, if $v(A) \in D$ and $v(A \rightarrow B) \in D$, then $v(B) \in D$. Assume also that $\mathcal{T}_{\mathfrak{M}}$ and $\vdash_{\mathfrak{M}}$ are subclassical.

Theorem 1 ([37]). $\mathcal{T}_{\mathfrak{M}}$ is maximal if for every formula $A \in \mathcal{T}_{\mathfrak{C}} \backslash \mathcal{T}_{\mathfrak{M}}$ there is a substitution $s^{\prime}$ such that $s^{\prime}(A) \rightarrow\left(p_{1} \rightarrow\left(\neg p_{1} \rightarrow p_{2}\right)\right) \in \mathcal{T}_{\mathfrak{M}}$. $\vdash_{\mathfrak{M}}$ is maximal if for every $\langle\mathcal{X}, A\rangle \in \vdash_{\mathfrak{C}} \backslash \vdash_{\mathfrak{M}}$ there is a substitution $s^{\prime \prime}$ such that $\left\{p_{1}, \neg p_{1}, s^{\prime \prime}(A)\right\} \vdash_{\mathfrak{M}} p_{2}$ and for every $X \in \mathcal{X}$ we have $\left\{p_{1}, \neg p_{1}\right\} \vdash_{\mathfrak{M}} s^{\prime \prime}(X)$.

This result represents a change of view when it comes to maximality: it focuses on formulas invalid (rejected) in $\mathfrak{C}$, including $E C Q$. In terms of previous research, the method we have been constantly referring to comprised of the above condition, stated in the form similar to Theorem 1, and bespoke substitutions (defined using the insights of [40]), each time

7 This means that there are formulas $A, B$ such that $A \rightarrow(\neg A \rightarrow B) \notin \mathcal{T}_{\mathfrak{M}}$. One can take issue with this definition as being too broad. After all, it is conceivable that, say, the schema $\neg A \rightarrow(A \rightarrow B) \in \mathcal{T}_{\mathfrak{M}}$ (or, at least the formula $\neg p_{1} \rightarrow\left(p_{1} \rightarrow p_{2}\right)$ is valid in $\mathfrak{M})$, which, so the thought goes, makes such logic undeserving of the moniker paraconsistent. We wish to sidestep the debate on what constitutes a bona fide paraconsistent logic: let us just note that in case of the logics considered by us in this article, this problem does not arise (details follow). See also [10] for a discussion of a curious feature of Sette's logic (we come back to this logic at a later of the article). 
crafted to fit the specific logic that was dealt with (see [34, 37]). Our approach extends this by showing, in essence, how a group of substitutions can be applied to a greater number of logics, thus generalising the scope of the method.

First, we showcase the method on a particular example of a threevalued logic.

Definition. Let $\mathfrak{D} \mathfrak{K}=\left\langle D K, D, \neg_{\mathfrak{D} \mathfrak{K}}, \rightarrow_{\mathfrak{D} \mathfrak{K}}\right\rangle$ be a logical matrix, where $D K=\{1,0,0.5\}, D=\{1,0.5\}$ and the interpretation for the connective symbols is provided by means of the following tables:

\begin{tabular}{c|c}
$A$ & $\neg \mathfrak{D} \mathfrak{K} A$ \\
\hline 1 & 0 \\
\hline 0 & 1 \\
\hline 0.5 & 0.5
\end{tabular}

\begin{tabular}{c|c|c|c}
$\rightarrow_{\mathfrak{D} \mathfrak{K}}$ & 1 & 0 & 0.5 \\
\hline 1 & 1 & 0 & 0.5 \\
\hline 0 & 1 & 1 & 1 \\
\hline 0.5 & 1 & 0 & 0.5
\end{tabular}

When a formula $A$ is valid in $\mathfrak{D} \mathfrak{K}$ we simply write $\models A$ without any subscripts.

\subsection{Basic properties}

Lemma 1. $\mathcal{T}_{\mathfrak{D} \mathfrak{K}}$ is paraconsistent.

Proof. Consider $p_{1} \rightarrow\left(\neg p_{1} \rightarrow p_{2}\right)$ and a valuation $v$, such that $v\left(p_{1}\right)=$ 0.5 and $v\left(p_{2}\right)=0$.

LEMma 2. $\vdash_{\mathfrak{D} \mathfrak{K}}$ is paraconsistent.

Proof. Consider $\left\langle\left\{p_{1}, \neg p_{1}\right\}, p_{2}\right\rangle$ and a valuation $v$, such that $v\left(p_{1}\right)=$ 0.5 and $v\left(p_{2}\right)=0$.

Let $A$ be a formula, if $v \in \mathcal{V}_{\mathfrak{D} \mathfrak{K}}$ is such that $v(A) \in\{0,1\}$, then $v(\neg A) \in\{0,1\}$ and for all $v^{\prime} \in \mathcal{V}_{\mathfrak{C}}$ such that $v(A)=v^{\prime}(A)$ we have $v(\neg A)=v^{\prime}(\neg A)$. Similarly, let $A$ and $B$ be formulas, if $v \in \mathcal{V}_{\mathfrak{D} \mathfrak{K}}$ is such that $v(A)$ and $v(B) \in\{0,1\}$, then $v(A \rightarrow B) \in\{0,1\}$ and for all $v^{\prime} \in \mathcal{V}_{\mathfrak{C}}$ such that $v(A)=v^{\prime}(A)$ and $v(B)=v^{\prime}(B)$ we have $v(A \rightarrow B)=v^{\prime}(A \rightarrow$ $B)$. From this and the definition of $v^{\prime} \in \mathcal{V}_{\mathfrak{C}}$ we see that $\mathcal{V}_{\mathfrak{C}} \subset \mathcal{V}_{\mathfrak{D} \mathfrak{K}}$. Since $\mathfrak{C}$ is a submatrix of $\mathfrak{D} \mathfrak{K}$, we easily obtain the following result.

Lemma $3 . \mathcal{T}_{\mathfrak{D} \mathfrak{K}}$ and $\vdash_{\mathfrak{D} \mathfrak{K}}$ are subclassical.

$\mathfrak{D} \mathfrak{K}$ also has the property that modus ponens is a valid rule of inference there. In order to see this, assume $A, B$ are formulas and $\mathcal{X}$ is 
a set of formulas. The following lemma ensures that if $\mathcal{X} \vdash_{\mathfrak{D} \mathfrak{K}} A$ and $\mathcal{X} \vdash_{\mathfrak{D} \mathfrak{K}} A \rightarrow B$, then $\mathcal{X} \vdash_{\mathfrak{D} \mathfrak{K}} B$.

Lemma 4. For any formulas $A, B$ and any valuation $v$, if $v(A) \in D$ and $v(A \rightarrow B) \in D$, then $v(B) \in D$.

Proof. Clearly $v(B) \in D$ as otherwise $v(A \rightarrow B) \notin D$ owing to the interpretation of implication, contradicting the assumption.

All this means that both $\mathcal{T}_{\mathfrak{D} \mathfrak{K}}$ and $\vdash_{\mathfrak{D} \mathfrak{K}}$ satisfy the conditions of Theorem 1, hence we can apply it in order to show what follows. We do this in two turns: first for $\mathcal{T}_{\mathfrak{D} \mathfrak{K}}$, then for $\vdash_{\mathfrak{D} \mathfrak{K}}$ in each case referring only to a fragment of Theorem 1. We have decided to keep the naming conventions for the different substitutions used in Theorem 1, to make it clear which part of the theorem is being referred to.

Lemma 5. $\mathcal{T}_{\mathfrak{D} \mathfrak{K}}$ is maximal.

Proof. By Theorem 1, $\mathcal{T}_{\mathfrak{D} \mathfrak{K}}$ is maximal if for every $A \in \mathcal{T}_{\mathfrak{C}} \backslash \mathcal{T}_{\mathfrak{D} \mathfrak{K}}$ there is a substitution $s^{\prime}$ such that $s^{\prime}(A) \rightarrow\left(p_{1} \rightarrow\left(\neg p_{1} \rightarrow p_{2}\right)\right) \in T_{\mathfrak{D} \mathfrak{K}}$. Consider a valuation $v$ such that $v(A)=0$ (such a valuation must exist). Now, for every formula $B$ define a substitution $s^{\prime}(B)$ as follows. For every $b \in \mathcal{S}_{B}$, let $s^{\prime}(b)=\neg\left(p_{1} \rightarrow\left(\neg p_{1} \rightarrow p_{2}\right)\right)$ if $v(b)=1 ; s^{\prime}(b)=p_{1} \rightarrow\left(\neg p_{1} \rightarrow p_{2}\right)$, if $v(b)=0$ and $s(b)=p_{1}$, if $v(b)=0.5$. We claim that $s^{\prime}(A) \rightarrow\left(p_{1} \rightarrow\right.$ $\left.\left(\neg p_{1} \rightarrow p_{2}\right)\right) \in T_{\mathfrak{D} \mathfrak{K}}$. Assume, for proof by contradiction, that this is not the case. Then there is a valuation $v^{\prime}$ such that $v^{\prime}\left(s^{\prime}(A)\right) \in D$ and $v^{\prime}\left(p_{1} \rightarrow\left(\neg p_{1} \rightarrow p_{2}\right)\right)=0$. In such a case, for all formulas $s^{\prime}(b)$ in $s^{\prime}(B)$ we have that $v^{\prime}\left(s^{\prime}(b)\right)=v(b)$, hence for every formula $v^{\prime}\left(s^{\prime}(B)\right)=v(B)$. In particular $v^{\prime}\left(s^{\prime}(A)\right)=v(A)=0$. A contradiction.

Lemma $6 . \vdash_{\mathfrak{D} \mathfrak{K}}$ is maximal.

Proof. By Theorem 1, $\vdash_{\mathfrak{D} \mathfrak{K}}$ is maximal if for every $\langle\mathcal{X}, A\rangle \in \vdash_{\mathfrak{C}} \backslash \vdash_{\mathfrak{D} \mathfrak{K}}$ there is a substitution $s^{\prime \prime}$ such that $\left\{p_{1}, \neg p_{1}, s^{\prime \prime}(A)\right\} \vdash_{\mathfrak{D} \mathfrak{K}} p_{2}$ and for every $X \in \mathcal{X}$ we have $\left\{p_{1}, \neg p_{1}\right\} \vdash_{\mathfrak{D} \mathfrak{K}} s^{\prime \prime}(X)$. Consider a valuation $v$ such that $v(\mathcal{X}) \in D$ and $v(A)=0$ (such a valuation must exist). Now, for every formula $B$ define a substitution $s^{\prime \prime}(B)$ as follows. For every $b \in \mathcal{S}_{B}$, let $s^{\prime \prime}(b)=p_{2} \rightarrow p_{2}$ if $v(b)=1 ; s^{\prime \prime}(b)=\neg\left(p_{2} \rightarrow p_{2}\right)$, if $v(b)=0$ and $s^{\prime \prime}(b)=$ $p_{1}$, if $v(b)=0.5$. We claim that $\left\{p_{1}, \neg p_{1}, s^{\prime \prime}(A)\right\} \vdash_{\mathfrak{D} \mathfrak{K}} p_{2}$. Assume, for proof by contradiction, that this is not the case. Then there is a valuation $v^{\prime}$ such that $v^{\prime}\left(p_{2}\right)=0, v^{\prime}\left(p_{1}\right)=0.5$ and $v^{\prime}\left(s^{\prime \prime}(A)\right) \in D$. In such a case, for all formulas $s^{\prime \prime}(b)$ in $s^{\prime \prime}(B)$ we have that $v^{\prime}\left(s^{\prime \prime}(b)\right)=v(b)$, hence for 
every formula $v^{\prime}\left(s^{\prime \prime}(B)\right)=v(B)$. In particular $v^{\prime}\left(s^{\prime \prime}(A)\right)=v(A)=0$. A contradiction. Furthermore, we claim that for every $X \in \mathcal{X}$ we have $\left\{p_{1}, \neg p_{1}\right\} \vdash_{\mathfrak{D} \mathfrak{K}} s^{\prime \prime}(X)$. Consider $v^{\prime \prime}$ such that $v^{\prime \prime}\left(p_{1}\right)=0.5$ (this is the only interesting case). If $v^{\prime \prime}\left(p_{2}\right)=0.5$, then for all formulas $s^{\prime \prime}(b)$ in $s^{\prime \prime}(B)$ we have that $v^{\prime \prime}\left(s^{\prime \prime}(b)\right)=0.5$ (by the way $s^{\prime \prime}$ is defined and given the particular interpretation of connectives), hence for every formula $v^{\prime \prime}\left(s^{\prime \prime}(B)\right)=0.5$ (this is shown by an easy induction; the focal point here is, again, the interpretation of connectives in the case when one has to take into account only the value 0.5 in an evaluation of a formula). In particular $v^{\prime \prime}\left(s^{\prime \prime}(X)\right)=0.5$. If $v^{\prime \prime}\left(p_{2}\right) \in\{1,0\}$, then for all formulas $s^{\prime \prime}(b)$ in $s^{\prime \prime}(B)$ we have that $v^{\prime \prime}\left(s^{\prime \prime}(b)\right)=v(b)$, hence for every formula $v^{\prime \prime}\left(s^{\prime \prime}(B)\right)=v(B)$. In particular $v^{\prime \prime}\left(s^{\prime \prime}(X)\right)=v(X) \in D$.

The method allows for a unified approach to searching for maximal subclassical paraconsistent logics. We emphasise that the proofs essentially boil down to finding an appropriate substitution.

The papers $[34,37]$ show how the method can be applied in the case of a number of well-known paraconsistent logics, including CAR, each time providing a substitution that works in a given setting. One can easily provide similar results for Sette's well-known maximal paraconsistent logic (for a description see, e.g., $[28,29]$ ). Let $\mathfrak{S T}=\left\langle S T, D, \neg \mathfrak{S T}, \rightarrow_{\mathfrak{S T}}\right.$ $>$ be a logical matrix, where $S T=\{1,0,0.5\}, D=\{1,0.5\}$ and the interpretation for the connective symbols is provided by means of the following tables:

\begin{tabular}{c|c}
$A$ & $\neg \mathfrak{S T} A$ \\
\hline 1 & 0 \\
\hline 0 & 1 \\
\hline 0.5 & 1
\end{tabular}

\begin{tabular}{c|c|c|c}
$\rightarrow_{\mathfrak{S T}}$ & 1 & 0 & 0.5 \\
\hline 1 & 1 & 0 & 1 \\
\hline 0 & 1 & 1 & 1 \\
\hline 0.5 & 1 & 0 & 1
\end{tabular}

Note that $\mathfrak{S T}$ satisfies the assumptions of Theorem 1. We can easily modify the proof of Lemma 5 to obtain the following.

Lemma 7. $\mathcal{T}_{\mathfrak{S T}}$ is maximal.

Proof. It is enough to modify the substitution $s^{\prime}(B)$ for every formula $B$ presented in Lemma 5 as follows. For every $b \in \mathcal{S}_{B}$, let $s^{\prime}(b)=\neg p_{1}$ if $v(b)=1 ; s^{\prime}(b)=p_{1} \rightarrow\left(\neg p_{1} \rightarrow p_{2}\right)$, if $v(b)=0$ and $s(b)=p_{1}$, if $v(b)=0.5$.

We also have the following result.

LEMma $8 . \vdash_{\mathfrak{S T}}$ is maximal. 
Proof. Everything proceeds analogously to the proof of Lemma 6 but in the part of the proof where one shows that for any $X \in \mathcal{X}$ we have $\left\{p_{1}, \neg p_{1}\right\} \vdash_{\mathfrak{D} \mathfrak{K}} s^{\prime \prime}(X)$ the difference in case of $v^{\prime \prime}\left(p_{2}\right)=0.5$ is that we obtain for any $B$ that $v^{\prime \prime}\left(s^{\prime \prime}(B)\right)=v(B)$, leading to contradiction.

To show how the method can be easily applied in other settings, for our final example we have chosen a lesser-known logic. It was developed in [15] and [16] in order to study mathematical theories with conditionally defined terms. Let $\mathfrak{H} \mathfrak{Z}=\left\langle H Z, D, \neg_{\mathfrak{H} \mathfrak{Z}}, \rightarrow_{\mathfrak{H} \mathfrak{Z}}\right\rangle$ be a logical matrix ${ }^{8}$ where $H Z=\{1,0,0.5\}, D=\{1,0.5\}$ and the interpretation for the connective symbols is provided by means of the following tables:

\begin{tabular}{c|c}
$A$ & $\neg_{\mathfrak{H} \mathfrak{Z}} A$ \\
\hline 1 & 0 \\
\hline 0 & 1 \\
\hline 0.5 & 0.5
\end{tabular}

\begin{tabular}{c|c|c|c}
$\rightarrow_{\mathfrak{H Z}}$ & 1 & 0 & 0.5 \\
\hline 1 & 1 & 0 & 0 \\
\hline 0 & 1 & 1 & 1 \\
\hline 0.5 & 1 & 0 & 0.5
\end{tabular}

Note that $\mathfrak{H} \mathfrak{Z}$ satisfies the assumptions of Theorem 1. We have the following:

Lemma 9. $\mathcal{T}_{\mathfrak{H} \mathfrak{Z}}$ is maximal.

Proof. We need to modify the substitution $s^{\prime}(B)$ for every formula $B$ presented in Lemma 5 as follows. For every $b \in \mathcal{S}_{B}$, let $s^{\prime}(b)=p_{2} \rightarrow p_{2}$ if $v(b)=1 ; s^{\prime}(b)=\neg\left(p_{2} \rightarrow p_{2}\right)$, if $v(b)=0$ and $s(b)=p_{1}$, if $v(b)=0.5$. In this case, however, we need to look closer into the valuations that make $s^{\prime}(A) \rightarrow\left(p_{1} \rightarrow\left(\neg p_{1} \rightarrow p_{2}\right)\right)$ false: $v_{1}$, where $v_{1}\left(s^{\prime}(A)\right)=1, v_{1}\left(p_{1} \rightarrow\right.$ $\left.\left(\neg p_{1} \rightarrow p_{2}\right)\right)=0$; $v_{2}$, where $v_{2}\left(s^{\prime}(A)\right)=0.5, v_{2}\left(p_{1} \rightarrow\left(\neg p_{1} \rightarrow p_{2}\right)\right)=0$ and crucially $v_{3}$, where $v_{3}\left(s^{\prime}(A)\right)=1, v_{3}\left(p_{1} \rightarrow\left(\neg p_{1} \rightarrow p_{2}\right)\right)=0.5$. We need to ensure that in all the cases we arrive at a contradiction. The cases of $v_{1}$ and $v_{2}$ are straightforward. In case of $v_{3}$, note that $v_{3}\left(p_{1} \rightarrow\left(\neg p_{1} \rightarrow\right.\right.$ $\left.\left.p_{2}\right)\right)=0.5$, when $v_{3}\left(p_{1}\right)=v_{3}\left(p_{2}\right)=0.5$, but then the way substitution is

8 Note that in this case, there were originally three, not two, primitive connectives but this is unimportant for the method at hand. The remaining connective $\wedge_{\mathfrak{H} \mathfrak{Z}}$ was originally defined as follows:

\begin{tabular}{c|c|c|c}
$\wedge_{\mathfrak{H} \mathfrak{Z}}$ & 1 & 0 & 0.5 \\
\hline 1 & 1 & 0 & 0.5 \\
\hline 0 & 0 & 0 & 0.5 \\
\hline 0.5 & 0.5 & 0.5 & 0.5
\end{tabular}

We emphatically do not use this definition (see also the section on computational analysis). 
defined, for any formula $B$ we have that $v_{3}\left(s^{\prime}(B)\right)=0.5$. This, obviously, includes $v_{3}\left(s^{\prime}(A)\right)=0.5$, contradicting the assumption.

We also easily obtain the following result.

Lemma $10 . \vdash_{\mathfrak{H} \mathfrak{Z}}$ is maximal.

Proof. Everything proceeds as in the proof of Lemma 6 .

The paper [26] shows maximality of $\vdash_{\mathfrak{H} \mathfrak{Z}}$ but uses advanced algebraic methods. Also, in contrast to the presented method, the proofs are much more specific to this particular logic and, arguably, might not lend themselves to generalisations.

\section{Generalisation}

This section gives a detailed account of how the refutation method can be applied to a greater number of three-valued paraconsistent logics. To be sure, there is research covering a broader number of systems. The paper [2] is a comprehensive study of three-valued paraconsistent logics, including their axiomatisation. It also deals with maximality, showing that all the well-known three-valued paraconsistent $\operatorname{logics}^{9}$ are maximal. A similar result is contained in [3] but it is set in a broader context of nondeterministic matrices. Such results, however, rely on a more complex argument than the one presented here. In what follows we present a proof of maximality that covers a number of paraconsistent logics.

\subsection{Maximality}

For the remainder of this paragraph let $v$ be a valuation defined within the context of the analysed classes of matrices. Also, let $X \in\{1,0,0.5\}$. A simple calculation shows that there are $3^{3} \times 3^{9}$ possible three-valued logical matrices (taking into account the possible value arrangements of tables for negation and implication). Out of these, at least ${ }^{10} 3 \times 3^{5}$ are subclassical in both senses, as shown in the following tables:

9 It should be noted that due to various ways in which both maximality and paraconsistency can be defined, it is hard to directly compare these results to the ones presented in this article.

10 There might be matrices that define subclassical logics that do not satisfy this condition. 


\begin{tabular}{c|c}
$A$ & $\neg A$ \\
\hline 1 & 0 \\
\hline 0 & 1 \\
\hline 0.5 & $X$
\end{tabular}

\begin{tabular}{c|c|c|c}
$\rightarrow$ & 1 & 0 & 0.5 \\
\hline 1 & 1 & 0 & $X$ \\
\hline 0 & 1 & 1 & $X$ \\
\hline 0.5 & $X$ & $X$ & $X$
\end{tabular}

This property is denoted (sub1). For a matrix having the (sub1) property, $D$ can be either $\{1\}$ or $\{1,0.5\}$, thus potentially doubling the number of logics. Let (par 1 ) denote the property of a matrix of having a paraconsistent induced consequence relation. Note that a matrix has this property if $D$ is the two-element set and we have $v(\neg A) \in D$, for $v(A)=0.5$, yielding the number of potential logical matrices equal to $2 \times 3^{5}$. ${ }^{11}$ Note that if $v$ is such that $v(A) \in D$ and $v(B)=0$ we should have that $v(A \rightarrow B)=0$ in order to enable the use of Theorem 1 . This property is denoted $(m p)$. This further reduces the number of logical matrices to $2 \times 3^{4}$ (see below).

\begin{tabular}{c|c}
$A$ & $\neg A$ \\
\hline 1 & 0 \\
\hline 0 & 1 \\
\hline 0.5 & $1 / 0.5$
\end{tabular}

\begin{tabular}{c|c|c|c}
$\rightarrow$ & 1 & 0 & 0.5 \\
\hline 1 & 1 & 0 & $X$ \\
\hline 0 & 1 & 1 & $X$ \\
\hline 0.5 & $X$ & 0 & $X$
\end{tabular}

In fact, we are interested in a somehow stronger notion of subclassicality, where both distinguished values behave analogously:

\begin{tabular}{c|c|c|c}
$\rightarrow$ & 1 & 0 & 0.5 \\
\hline 1 & 1 & 0 & $1 / 0.5$ \\
\hline 0 & 1 & 1 & $1 / 0.5$ \\
\hline 0.5 & $1 / 0.5$ & 0 & $1 / 0.5$
\end{tabular}

We denote this property (sub2). This reduces the number of logical matrices to $2 \times 2^{4}=32$. Another simple observation is that if a threevalued subclassical logical matrix has the property $(\mathrm{mp})$, then it has a paraconsistent theory (the property of having a paraconsistent theory is

11 As has been pointed out to us, this is not a necessary condition. However, our approach to generalisation is admittedly simplistic, which is perhaps necessary at this early stage. We try to look for certain easily-observable patterns that lend themselves well to be used in the context of our method, focusing on how implication is being defined. The case of $\mathfrak{H} \mathfrak{Z}$ shows that maximality can also be a result of a more complex interplay between the definitions of connectives. Identifying such complex patterns that yield maximality of logical matrices from a given class requires additional research and goes beyond the scope of this paper. 
from now on denoted as (par2)). To see this note that in such case the formula $p_{1} \rightarrow\left(\neg p_{1} \rightarrow p_{2}\right)$ is falsified by the valuation assigning 0.5 to $p_{1}$ and 0 to $p_{2}$. It does not matter whether the considered matrix satisfies the (sub2) property or not. We, however, take only those logical matrices that do. In this context, note that the above observation holds independently of the way, in which negation is defined. Thus, the total number of logics we potentially take into consideration does not change. ${ }^{12}$

\subsection{Maximality of theory}

Theorem 1 allows one to draw this simple, yet surprising, conclusion.

Theorem 2. If $\mathfrak{M}$ is a logical matrix having both (sub2) and ( $m p)$ properties, then $\mathcal{T}_{\mathfrak{M}}$ is maximal.

Proof. The fact that $\mathfrak{M}$ has a maximal theory relies on a modification of the proof of Lemma 5 and the fact that $\mathfrak{M}$ also satisfies (par2), as described above. Assume all the notational conventions made in the proof of that lemma. What is required there is a substitution $s^{\prime}$ such that it fixes the values of formulas in relation to the specified valuation $v$, resulting in contradiction. There are many ways of accomplishing this task but arguably the easiest one uses the formula $p_{1} \rightarrow\left(\neg p_{1} \rightarrow p_{2}\right)$. We use the fact that in logical matrices with paraconsistent theory such a formula is an instance of $E C Q$. In particular, for $\mathfrak{M}$, there is a valuation $v^{\prime}$ such that $v^{\prime}\left(p_{1} \rightarrow\left(\neg p_{1} \rightarrow p_{2}\right)\right)=0$. By $(s u b 2)$, we have to consider $v^{\prime}$ such that $v^{\prime}\left(p_{1}\right)=0.5$ and $v^{\prime}\left(p_{2}\right)=0$. Then, the required substitution can be defined exactly as in the proof of Lemma 5. For every $b \in \mathcal{S}_{B}$, let $s^{\prime}(b)=\neg\left(p_{1} \rightarrow\left(\neg p_{1} \rightarrow p_{2}\right)\right)$ if $v(b)=1 ; s^{\prime}(b)=p_{1} \rightarrow\left(\neg p_{1} \rightarrow p_{2}\right)$, if $v(b)=0$ and $s(b)=p_{1}$, if $v(b)=0.5$. This fixes the values in the required way for proof by contradiction, thus proving that $\mathcal{T}_{\mathfrak{M}}$ is maximal.

Thus, we see that two of the examples we have given earlier, namely $\mathfrak{D} \mathfrak{K}$ and $\mathfrak{S T}$ do fall into this general category, with $\mathfrak{H} \mathfrak{Z}$ being something of an outlier. It is a matter of simple observation that for matrices having both $(s u b 2)$ and $(m p)$ properties we have that $\neg p_{1} \rightarrow\left(p_{1} \rightarrow p_{2}\right)$ is not valid. Hence the considered logics meet even the narrower criteria for paraconsistency, as mentioned at the beginning of this article.

12 Other paraconsistent, subclassical logics, arrived at by different means lie outside the scope of this article. 


\subsection{Maximality of consequence}

Showing that three-valued logical matrices have maximal consequence relation is more involved. First, we need to distinguish between several types of logical matrices. Let $\mathbf{M}_{1}$ be a class of logical matrices with the following constraints on negation and implication.

\begin{tabular}{c|c}
$A$ & $\neg A$ \\
\hline 1 & 0 \\
\hline 0 & 1 \\
\hline 0.5 & 0.5
\end{tabular}

\begin{tabular}{c|c|c|c}
$\rightarrow$ & 1 & 0 & 0.5 \\
\hline 1 & 1 & 0 & $X$ \\
\hline 0 & 1 & 1 & $X$ \\
\hline 0.5 & $X$ & 0 & $1 / 0.5$
\end{tabular}

Let $\mathbf{M}_{2}$ be a class of logical matrices with the following constraints on negation and implication.

\begin{tabular}{c|c}
$A$ & $\neg A$ \\
\hline 1 & 0 \\
\hline 0 & 1 \\
\hline 0.5 & 1
\end{tabular}

\begin{tabular}{c|c|c|c}
$\rightarrow$ & 1 & 0 & 0.5 \\
\hline 1 & 1 & 0 & $1 / 0.5$ \\
\hline 0 & 1 & 1 & $X$ \\
\hline 0.5 & $1 / 0.5$ & 0 & 0.5
\end{tabular}

Finally, let $\mathbf{M}_{3}$ be a class of logical matrices with the following constraints on negation and implication.

\begin{tabular}{c|c}
$A$ & $\neg A$ \\
\hline 1 & 0 \\
\hline 0 & 1 \\
\hline 0.5 & 1
\end{tabular}

\begin{tabular}{c|c|c|c}
$\rightarrow$ & 1 & 0 & 0.5 \\
\hline 1 & 1 & 0 & $X$ \\
\hline 0 & 1 & 1 & $X$ \\
\hline 0.5 & $X$ & 0 & 1
\end{tabular}

Note that the members of all the three defined classes satisfy condition (par 1$)$.

Theorem 3. If $\mathfrak{M} \in \mathbf{M}_{i}(1 \leqslant i \leqslant 3)$, then $\vdash_{\mathfrak{M}}$ is maximal.

Proof. The proof also relies on a substitution that fixes the values of formulas in relation to the specified valuation $v$, this time as defined for Lemma 6 . We use the fact that $\vdash_{\mathfrak{M}}$ is paraconsistent. In particular $\left\langle\left\{p_{1}, \neg p_{1}\right\}, p_{2}\right\rangle$ is not in $\vdash_{\mathfrak{M}}$. Consider a tuple of $\langle\mathcal{X}, A\rangle \in \vdash_{\mathfrak{C}} \backslash \vdash_{\mathfrak{M}}$. The required substitution is the same as in the proof of Lemma 6: for every $b \in \mathcal{S}_{B}$, let $s^{\prime \prime}(b)=p_{2} \rightarrow p_{2}$ if $v(b)=1 ; s^{\prime \prime}(b)=\neg\left(p_{2} \rightarrow p_{2}\right)$, if $v(b)=0$ and $s^{\prime \prime}(b)=p_{1}$, if $v(b)=0.5$. This fixes the values in reference to $v$ in the required way and by the same reasoning as in the proof of Lemma 6 we get $\left\{p_{1}, \neg p_{1}, s^{\prime \prime}(A)\right\} \vdash_{\mathfrak{M}} p_{2}$. To show that for every $X \in \mathcal{X}$ we have $\left\{p_{1}, \neg p_{1}\right\} \vdash_{\mathfrak{M}} s^{\prime \prime}(X)$ we focus on $v^{\prime \prime}$ such that $v^{\prime \prime}\left(p_{1}\right)=0.5$. Note that if 
$v^{\prime \prime}\left(p_{2}\right) \in\{1,0\}$, then the value of all the substituted formulas is fixed in reference to $v$ as in the proof of Lemma 6 , hence we focus on $v^{\prime \prime}\left(p_{2}\right)=0.5$. We now have several cases to consider:

1. If $\mathfrak{M} \in \mathbf{M}_{1}$, then the value of all substituted formulas is fixed to be 0.5 or is fixed in reference to $v$, as in the proof of Lemma 6 .

2. If $\mathfrak{M} \in \mathbf{M}_{2}$, then the value of all substituted formulas is fixed to be either 1 or 0.5 .

3. If $\mathfrak{M} \in \mathbf{M}_{3}$, then the value of all substituted formulas is fixed in reference to $v$ as in the proof of Lemma 6 .

In all the considered cases we obtain $v^{\prime \prime}\left(s^{\prime \prime}(X)\right) \in D$, as required.

\section{Computational analysis}

We wish to experimentally explore the properties of the $2 \times 16$ matrices having both maximal theory and consequence relations and see how these compare to the rest in that setting. We propose a more practicallyoriented way of measuring how "close" these are to the classical one. To that end, we have designed a validity checker ${ }^{13}$ for the three-valued subclassical matrices. It works by creating a truth-table validity check for a given set of formulas having at most three variables. This validity check can be done on all the subclassical matrices; however, we decided to limit our attention to those matrices that have both (sub1) and ( $m p)$ properties, thus potentially falling within the scope of Theorem 1. We wished to experimentally check whether there are practical indications that there are maximal subclassical logical matrices that were not captured by the theoretical analyses using the method we have employed but which could be perhaps identified using a modified version of it. Hence, we took the following, standard, list of - independent-axioms for the classical propositional logic and checked which of these are valid in a given subclassical logical matrix.

1. $p_{1} \rightarrow\left(p_{2} \rightarrow p_{1}\right)$

2. $\left[p_{1} \rightarrow\left(p_{2} \rightarrow p_{3}\right)\right] \rightarrow\left[\left(p_{1} \rightarrow p_{2}\right) \rightarrow\left(p_{1} \rightarrow p_{3}\right)\right]$

3. $\quad\left(p_{1} \rightarrow p_{2}\right) \rightarrow\left(\neg p_{2} \rightarrow \neg p_{1}\right)$

13 Very limited in terms of applications, essentially custom-built to perform the job at hand. It can be, however, easily extended to perform other, similar functions. The code is available at the following http://www.logic.ifil.uz.zgora.pl/code/ 3val-validity-check.py. 


$$
\begin{aligned}
\text { 4. } & \neg \neg p_{1} \rightarrow p_{1} \\
\text { 5. } & p_{1} \rightarrow \neg \neg p_{1} \\
\text { 6. } & p_{1} \wedge p_{2} \rightarrow p_{1} \\
\text { 7. } & p_{1} \wedge p_{2} \rightarrow p_{2} \\
\text { 8. } & \left(p_{1} \rightarrow p_{2}\right) \rightarrow\left[\left(p_{1} \rightarrow p_{3}\right) \rightarrow\left(p_{1} \rightarrow p_{2} \wedge p_{3}\right)\right] \\
\text { 9. } & p_{1} \rightarrow\left(p_{2} \vee p_{1}\right) \\
\text { 10. } & p_{2} \rightarrow\left(p_{2} \vee p_{1}\right) \\
\text { 11. } & \left(p_{1} \rightarrow p_{3}\right) \rightarrow\left[\left(p_{2} \rightarrow p_{3}\right) \rightarrow\left(p_{1} \vee p_{2} \rightarrow p_{3}\right)\right] \\
\text { 12. } & \left(p_{1} \leftrightarrow p_{2}\right) \rightarrow\left(p_{2} \rightarrow p_{1}\right) \\
\text { 13. } & \left(p_{1} \leftrightarrow p_{2}\right) \rightarrow\left(p_{1} \rightarrow p_{2}\right) \\
\text { 14. } & \left(p_{1} \rightarrow p_{2}\right) \rightarrow\left[\left(p_{2} \rightarrow p_{1}\right) \rightarrow\left(p_{1} \leftrightarrow p_{2}\right)\right]
\end{aligned}
$$

Obviously, a decision had to be made regarding how the remaining connectives should be defined. We have assumed the following definitions:

$$
\begin{aligned}
& A \vee B:=\neg A \rightarrow B ; \\
& A \wedge B:=\neg(\neg A \vee \neg B) ; \\
& A \equiv B:=(A \rightarrow B) \wedge(B \rightarrow A) .
\end{aligned}
$$

All the 243 possible tables for implication used in defining subclassical logical matrices were enumerated and then only those satisfying the condition $(m p)$ were selected for further analysis (see Appendix A). Each of the tables for implication was used twice: once for each possible way of defining negation. Therefore, the numbering is doing double duty here: it is used to describe the specific table for implication as well as the resulting logical matrix (one table for implication can be paired with each of the two possible tables for negation, thus giving rise to $2 \times 243$ logical matrices). The matrices were assigned scores depending on the number of the above axioms they satisfied. For negation defined as $\neg 0.5=0.5$, the results were as follows:

$[(168,12),(4,11),(6,11),(166,11),(186,11),(222,10),(22,9),(24,9),(58,9),(60,9),(87,9)$, $(184,9),(220,9),(13,8),(240,8),(15,7),(85,7),(86,7),(114,7),(195,7),(5,6),(14,6)$, $(76,6),(78,6),(94,6),(95,6),(96,6),(105,6),(121,6),(122,6),(123,6),(238,6),(31,5)$, $(33,5),(40,5),(42,5),(112,5),(113,5),(141,5),(193,5),(213,5),(32,4),(41,4),(103,4)$, $(139,4),(157,4),(159,4),(175,4),(49,3),(51,3),(177,3),(231,3),(23,2),(67,2),(69,2)$, $(130,2),(132,2),(148,2),(150,2),(167,2),(185,2),(202,2),(204,2),(211,2),(212,2)$, $(229,2),(50,1),(59,1),(104,1),(140,1),(176,1),(194,1),(68,0),(77,0),(131,0),(149,0)$, $(158,0),(203,0),(221,0),(230,0),(239,0)]$

Each pair represents a matrix number and the score obtained respectively. For example, 168, with the highest score represents our logical matrix $\mathfrak{D} \mathfrak{K}$, showing that in some way it stands out among the others. This is obviously heavily dependent on the way, in which we decide to define the remaining connectives: and it seems that at least for $\mathfrak{D} \mathfrak{K}$ the 
one we have chosen might be optimal. The runner-up, 4 uses the implication table as defined in $\mathfrak{S T}$ but with negation defined differently: it seems it is a powerful system nevertheless. All in all, the results confirm the strong position of the logical matrices identified in our theoretical analysis: in fact, the first 15 results comprise of 14 matrices covered in the proof of Theorem 4.2 and the remaining one (87) represents $\mathfrak{H} \mathfrak{Z}$, which, as mentioned earlier, does not quite fit into the picture in terms of generalisation. Remarkable!

For negation defined as $\neg 0.5=1$, the results were as follows:

$[(4,10),(58,10),(86,10),(95,10),(6,9),(22,9),(24,9),(60,9),(76,9),(78,9),(85,9)$, $(94,9),(166,9),(168,9),(184,9),(186,9),(220,9),(222,9),(238,9),(240,9)(5,8),(13,8)$, $(14,8),(87,8),(140,8),(103,7),(104,7),(105,7),(139,7),(141,7),(157,7),(158,7),(231,7)$, $(15,6),(23,6),(59,6),(96,6),(113,6),(122,6),(159,6),(175,6),(229,6),(67,5),(68,5)$, $(69,5),(77,5),(148,5),(149,5),(150,5),(167,5),(176,5),(177,5),(185,5),(221,5),(230,5)$, $(239,5),(32,4),(41,4),(112,4),(121,4),(40,3),(31,3),(130,2),(131,2),(114,1),(132,1)$, $(33,0),(42,0),(49,0),(50,0),(51,0),(123,0),(193,0),(194,0),(195,0),(202,0),(203,0)$, $(204,0),(211,0),(212,0),(213,0)]$

We see that the same sort of pattern emerges here but the landscape is more varied: most of the logical matrices identified by us as having both maximal theory and consequence relations are to be found among the top 20 results with some matrices trading places (4, representing $\mathfrak{S T}$ is now among the top-scored results). One notes that there are a number of other matrices entering the picture when negation is defined differently (e.g. 86, 95); also the two matrices satisfying both (sub2) and ( $m p)$ (e.g. 76 and 78) that were previously trailing behind, have now secured somehow more satisfying spots in the ranking. This new way of defining negation, however, seems to have a deteriorating effect on 87, with its position being noticeably weaker. But it seems safe to conclude that also in this case, generally speaking, the practical analysis confirmed the theoretical results (potentially opening new avenues of research as well).

We note in passing that this simple experiment also shows that when $\mathfrak{D} \mathfrak{K}, \mathfrak{S T}$ and $\mathfrak{H} \mathfrak{Z}$ are extended with the interpretations of additional connectives as above, these all give rise to different theories. There are a number of things one needs to be aware of at this point. (1) $\mathfrak{S T}$ is traditionally extended with connectives defined in a different way (see [28], [29]) and an initial computational analysis suggests that this way might indeed be optimal for it, yielding more axioms from the above list being satisfied. (2) The question whether the other described matrices, beyond the three examples we focused on, extended in the above way also define distinct theories is still open. In many cases, it should not be difficult to establish the answer by means of a simple (computer-aided) check but this goes beyond the scope of our work. 
Perhaps we should emphasise at this point that here we focus on identifying matrices that give rise to maximal theories and consequence relations, rather than on comparing the theories and relations that can be obtained via different matrices.

\section{Conclusions and further work}

Our main aim was to show how a certain refutation method can be generalised to cover a large number of paraconsistent logics in terms of proving their maximality. To tally up, we have identified 32 threevalued logics that are subclassical, paraconsistent and maximal in both senses distinguished in this article. Observe that the number of matrices having a paraconsistent consequence relation is much greater. A question suggests itself whether the remaining matrices can also be shown to have a maximal paraconsistent theory. It would also be interesting to precisely compare the presented results with the ones described in [2] and [3] and see how well the method lends itself to further generalisations. One can try and extend the use of this method even further, e.g. to fourvalued systems or, perhaps, show how it can be used in the most general setting of $n$-valued logics, where $n$ is any number, alternatively with some restrictions imposed on $n$. As for the experimental part, it would be interesting to further compare the analysed matrices and perform a more detailed research in terms of which formulas are valid in these depending on various ways of defining the remaining connectives. For example, the connectives for $\mathfrak{S T}$ have been originally defined differently than we have done in this article and this does make a difference in terms of the formulas valid in the resulting logic. It would also be interesting to further compare $\mathfrak{S T}$ and $\mathfrak{D} \mathfrak{K}$, both from the theoretical and practical points of view. So far, we have seen that the theoretical part shaped the practical investigations, but it might well be the other way round: the experimental results might, in turn, inform a more theoretical analysis. Having some sort of practical confirmation of the strength of a given logical matrix, one could focus on such systems in hope proving their maximality. We see that given the simplicity of the method, it lends itself well to all sorts of applications in computer science. Hence, another interesting avenue of research would be to design, on the basis of the formal description of the method, a computer program that performs the search for maximal paraconsistent logics of three, four or more values. 


\section{A. Appendix}

A look-up table for all the possible implication matrices satisfying (sub1). Each list to be read as follows: [matrix-number, a, b, c, d,e], where the letters relate to the positions in the actual tables as indicated below.

\begin{tabular}{c|c|c|c}
$\rightarrow$ & 1 & 0 & 0.5 \\
\hline 1 & 1 & 0 & $\mathrm{a}$ \\
\hline 0 & 1 & 1 & $\mathrm{~b}$ \\
\hline 0.5 & $\mathrm{c}$ & $\mathrm{d}$ & $\mathrm{e}$
\end{tabular}

$[1,1,1,1,1,1],[2,1,1,1,1,0],[3,1,1,1,1,0.5],[4,1,1,1,0,1]$,

$[5,1,1,1,0,0],[6,1,1,1,0,0.5],[7,1,1,1,0.5,1],[8,1,1,1,0.5,0]$,

$[9,1,1,1,0.5,0.5],[10,1,1,0,1,1],[11,1,1,0,1,0],[12,1,1,0,1,0.5]$,

$[13,1,1,0,0,1],[14,1,1,0,0,0],[15,1,1,0,0,0.5],[16,1,1,0,0.5,1]$,

$[17,1,1,0,0.5,0],[18,1,1,0,0.5,0.5],[19,1,1,0.5,1,1],[20,1,1,0.5,1,0]$,

$[21,1,1,0.5,1,0.5],[22,1,1,0.5,0,1],[23,1,1,0.5,0,0],[24,1,1,0.5,0,0.5]$

$[25,1,1,0.5,0.5,1],[26,1,1,0.5,0.5,0],[27,1,1,0.5,0.5,0.5],[28,1,0,1,1,1]$,

$[29,1,0,1,1,0],[30,1,0,1,1,0.5],[31,1,0,1,0,1],[32,1,0,1,0,0]$,

$[33,1,0,1,0,0.5],[34,1,0,1,0.5,1],[35,1,0,1,0.5,0],[36,1,0,1,0.5,0.5]$,

$[37,1,0,0,1,1],[38,1,0,0,1,0],[39,1,0,0,1,0.5],[40,1,0,0,0,1]$,

$[41,1,0,0,0,0],[42,1,0,0,0,0.5],[43,1,0,0,0.5,1],[44,1,0,0,0.5,0]$,

$[45,1,0,0,0.5,0.5],[46,1,0,0.5,1,1],[47,1,0,0.5,1,0],[48,1,0,0.5,1,0.5]$,

$[49,1,0,0.5,0,1],[50,1,0,0.5,0,0],[51,1,0,0.5,0,0.5],[52,1,0,0.5,0.5,1]$,

$[53,1,0,0.5,0.5,0],[54,1,0,0.5,0.5,0.5],[55,1,0.5,1,1,1],[56,1,0.5,1,1,0]$,

$[57,1,0.5,1,1,0.5],[58,1,0.5,1,0,1],[59,1,0.5,1,0,0],[60,1,0.5,1,0,0.5]$,

$[61,1,0.5,1,0.5,1],[62,1,0.5,1,0.5,0],[63,1,0.5,1,0.5,0.5],[64,1,0.5,0,1,1]$,

$[65,1,0.5,0,1,0],[66,1,0.5,0,1,0.5],[67,1,0.5,0,0,1],[68,1,0.5,0,0,0]$,

$[69,1,0.5,0,0,0.5],[70,1,0.5,0,0.5,1],[71,1,0.5,0,0.5,0],[72,1,0.5,0,0.5,0.5]$,

$[73,1,0.5,0.5,1,1],[74,1,0.5,0.5,1,0],[75,1,0.5,0.5,1,0.5],[76,1,0.5,0.5,0,1]$,

$[77,1,0.5,0.5,0,0],[78,1,0.5,0.5,0,0.5],[79,1,0.5,0.5,0.5,1],[80,1,0.5,0.5,0.5,0]$,

$[81,1,0.5,0.5,0.5,0.5],[82,0,1,1,1,1],[83,0,1,1,1,0],[84,0,1,1,1,0.5]$,

$[85,0,1,1,0,1],[86,0,1,1,0,0],[87,0,1,1,0,0.5],[88,0,1,1,0.5,1]$,

$[89,0,1,1,0.5,0],[90,0,1,1,0.5,0.5],[91,0,1,0,1,1],[92,0,1,0,1,0]$,

$[93,0,1,0,1,0.5],[94,0,1,0,0,1],[95,0,1,0,0,0],[96,0,1,0,0,0.5]$,

$[97,0,1,0,0.5,1],[98,0,1,0,0.5,0],[99,0,1,0,0.5,0.5],[100,0,1,0.5,1,1]$,

$[101,0,1,0.5,1,0],[102,0,1,0.5,1,0.5],[103,0,1,0.5,0,1],[104,0,1,0.5,0,0]$,

$[105,0,1,0.5,0,0.5],[106,0,1,0.5,0.5,1],[107,0,1,0.5,0.5,0],[108,0,1,0.5,0.5,0.5]$,

$[109,0,0,1,1,1],[110,0,0,1,1,0],[111,0,0,1,1,0.5],[112,0,0,1,0,1]$,

$[113,0,0,1,0,0],[114,0,0,1,0,0.5],[115,0,0,1,0.5,1],[116,0,0,1,0.5,0]$,

$[117,0,0,1,0.5,0.5],[118,0,0,0,1,1],[119,0,0,0,1,0],[120,0,0,0,1,0.5]$,

$[121,0,0,0,0,1],[122,0,0,0,0,0],[123,0,0,0,0,0.5],[124,0,0,0,0.5,1]$,

$[125,0,0,0,0.5,0],[126,0,0,0,0.5,0.5],[127,0,0,0.5,1,1],[128,0,0,0.5,1,0]$,

$[129,0,0,0.5,1,0.5],[130,0,0,0.5,0,1],[131,0,0,0.5,0,0],[132,0,0,0.5,0,0.5]$,

$[133,0,0,0.5,0.5,1],[134,0,0,0.5,0.5,0],[135,0,0,0.5,0.5,0.5],[136,0,0.5,1,1,1]$,

$[137,0,0.5,1,1,0],[138,0,0.5,1,1,0.5],[139,0,0.5,1,0,1],[140,0,0.5,1,0,0]$,

$[141,0,0.5,1,0,0.5],[142,0,0.5,1,0.5,1],[143,0,0.5,1,0.5,0],[144,0,0.5,1,0.5,0.5]$,

$[145,0,0.5,0,1,1],[146,0,0.5,0,1,0],[147,0,0.5,0,1,0.5],[148,0,0.5,0,0,1]$,

$[149,0,0.5,0,0,0],[150,0,0.5,0,0,0.5],[151,0,0.5,0,0.5,1],[152,0,0.5,0,0.5,0]$,

$[153,0,0.5,0,0.5,0.5],[154,0,0.5,0.5,1,1],[155,0,0.5,0.5,1,0],[156,0,0.5,0.5,1,0.5]$,

$[157,0,0.5,0.5,0,1],[158,0,0.5,0.5,0,0],[159,0,0.5,0.5,0,0.5],[160,0,0.5,0.5,0.5,1]$,

$[161,0,0.5,0.5,0.5,0],[162,0,0.5,0.5,0.5,0.5],[163,0.5,1,1,1,1],[164,0.5,1,1,1,0]$,

$[165,0.5,1,1,1,0.5],[166,0.5,1,1,0,1],[167,0.5,1,1,0,0],[168,0.5,1,1,0,0.5]$,

$[169,0.5,1,1,0.5,1],[170,0.5,1,1,0.5,0],[171,0.5,1,1,0.5,0.5],[172,0.5,1,0,1,1]$,

$[173,0.5,1,0,1,0],[174,0.5,1,0,1,0.5],[175,0.5,1,0,0,1],[176,0.5,1,0,0,0]$,

$[177,0.5,1,0,0,0.5],[178,0.5,1,0,0.5,1],[179,0.5,1,0,0.5,0],[180,0.5,1,0,0.5,0.5]$,

$[181,0.5,1,0.5,1,1],[182,0.5,1,0.5,1,0],[183,0.5,1,0.5,1,0.5],[184,0.5,1,0.5,0,1]$,

$[185,0.5,1,0.5,0,0],[186,0.5,1,0.5,0,0.5],[187,0.5,1,0.5,0.5,1],[188,0.5,1,0.5,0.5,0]$,

$[189,0.5,1,0.5,0.5,0.5],[190,0.5,0,1,1,1],[191,0.5,0,1,1,0],[192,0.5,0,1,1,0.5]$,

$[193,0.5,0,1,0,1],[194,0.5,0,1,0,0],[195,0.5,0,1,0,0.5],[196,0.5,0,1,0.5,1]$,

$[197,0.5,0,1,0.5,0],[198,0.5,0,1,0.5,0.5],[199,0.5,0,0,1,1],[200,0.5,0,0,1,0]$,

$[201,0.5,0,0,1,0.5],[202,0.5,0,0,0,1],[203,0.5,0,0,0,0],[204,0.5,0,0,0,0.5]$,

$[205,0.5,0,0,0.5,1],[206,0.5,0,0,0.5,0],[207,0.5,0,0,0.5,0.5],[208,0.5,0,0.5,1,1]$,

$[209,0.5,0,0.5,1,0],[210,0.5,0,0.5,1,0.5],[211,0.5,0,0.5,0,1],[212,0.5,0,0.5,0,0]$,

$[213,0.5,0,0.5,0,0.5],[214,0.5,0,0.5,0.5,1],[215,0.5,0,0.5,0.5,0],[216,0.5,0,0.5,0.5,0.5]$, 
$[217,0.5,0.5,1,1,1],[218,0.5,0.5,1,1,0],[219,0.5,0.5,1,1,0.5],[220,0.5,0.5,1,0,1]$, $[221,0.5,0.5,1,0,0],[222,0.5,0.5,1,0,0.5],[223,0.5,0.5,1,0.5,1],[224,0.5,0.5,1,0.5,0]$, $[225,0.5,0.5,1,0.5,0.5],[226,0.5,0.5,0,1,1],[227,0.5,0.5,0,1,0],[228,0.5,0.5,0,1,0.5]$, $[229,0.5,0.5,0,0,1],[230,0.5,0.5,0,0,0],[231,0.5,0.5,0,0,0.5],[232,0.5,0.5,0,0.5,1]$, $[233,0.5,0.5,0,0.5,0],[234,0.5,0.5,0,0.5,0.5],[235,0.5,0.5,0.5,1,1],[236,0.5,0.5,0.5,1,0]$, $[237,0.5,0.5,0.5,1,0.5],[238,0.5,0.5,0.5,0.1],[239,0.5,0.5,0.5,0,0],[240,0.5,0.5,0.5,0,0.5]$, $[241,0.5,0.5,0.5,0.5,1],[242,0.5,0.5,0.5,0.5,0],[243,0.5,0.5,0.5,0.5,0.5]$

Acknowledgment. I should like to express my gratitude to the anonymous referee, whose remarks allowed me to extend the paper, refine it and avoid many a mistake, including one that would have been very embarrassing indeed.

\section{References}

[1] Alves, E. H., "The first axiomatization of paraconsistent logic", Bulletin of the Section of Logic 21, 1 (1992): 19-20.

[2] Arieli, O., and A. Avron., "Three-valued paraconsistent propositional logics", pages 91-129 in J.-Y. Beziau, M. Chakraborty, and S. Dutta (eds.), New Directions in Paraconsistent Logic, Springer, 2015. DOI: 10.1007/ 978-81-322-2719-9_4

[3] Arieli, O., A. Avron, and A. Zamansky, "Maximally paraconsistent threevalued logics", pages 210-218 in F. Lin, U. Sattler, and M. Truszczynski (eds.), Proceedings of the Twelfth International Conference on the Principles of Knowledge Representation and Reasoning, The AAAI Press, Menlo Park, California, 2010.

[4] Arruda, A. I., "Aspects of the historical development of paraconsistent Logic", pages 99-130 in G. Priest, R. Roultey, and X. Norman (eds.), Paraconsistent Logic: Essays on the Inconsistent, Philosophia Verlag, 1989.

[5] Batens, D., "Paraconsistency and its relation to worldviews", Foundations of Science 3 (1999): 259-283.

[6] Batens, D., "A deneral characterization of adaptive logics", Logique et Analyse 173-175 (2002): 45-68.

[7] Batóg, T., Dwa paradygmaty matematyki, Wydawnictwo Naukowe UAM, Poznań, 2000.

[8] Caicedo, X., "A formal system for the non-theorems of the propositional calculus", Notre Dame Journal of Formal Logic 19, 1 (1978): 147-151. DOI: $10.1305 / \mathrm{ndjfl} / 1093888218$

[9] Carnap, R., Formalization of Logic, Harvard University Press, 1943.

[10] Ciuciura, J., "Paraconsistency and Sette's calculus P1", Logic and Logical Philosophy 24 (2015): 265-273. DOI: 10.12775/LLP.2015.003

[11] Gabbay, D.M., and A.S. d'Avila Garcez, "Logical modes of attack in argumentation networks", Studia Logica 93, 2-3 (2009): 199-230. DOI: $10.1007 / \mathrm{s} 11225-009-9216-\mathrm{z}$ 
[12] Ganeri, J., "Jaina logic and the philosophical basis for pluralism", History and Philosophy of Logic 23, 4 (2002): 267-281. DOI: 10.1080/ 0144534021000051505

[13] Goranko, V., "Proving unprovability in some normal modal logics", Bulletin of the Section of Logic 20, 1 (1991): 23-29.

[14] Goranko, V., "Refutation systems in modal logics", Studia Logica 53, 2 (1994): 299-324. DOI: $10.1007 / \mathrm{BF} 01054714$

[15] Hałkowska, K., "A note on matrices for systems of nonsense-logics", Studia Logica 48, 4 (1989): 461-464. DOI: 10.1007/BF00370200

[16] Hałkowska, K., and A. Zając, "O pewnym trójwartościowym systemie rachunku zdań", Acta Universitatis Wratislaviensis. Prace Filozoficzne. Logika, 13 (1988): 40-51.

[17] Jaśkowski, S., "A propositional calculus for inconsistent deductive systems", Logic and Logical Philosophy 7 (1999): 35-56. DOI: 10.12775/ LLP. 1999.003

[18] Łukasiewicz, J., O zasadzie sprzeczności u Arystotelesa, Państwowe Wydawnictwo Naukowe, Warszawa, 1956.

[19] Łukasiewicz, J., Aristotle's Syllogistic from the Standpoint of Modern Formal Logic, Clarendon Press, Oxford, 1957.

[20] Łukasiewicz, J., "Two-valued logic" (1921), pages 89-109 in L. Borkowski (ed.), Jan Lukasiewicz. Selected Works, North Holland Publishing Company, Polish Scientific Publishers, 1970.

[21] Pietryga, A., Status zasady sprzeczności w świetle logiki wspótczesnej, Aureus, 2004.

[22] Poczobut, R., Spór o zasadę niesprzeczności, Towarzystwo Naukowe KUL, 2000.

[23] Priest, G., In Contradiction, Kluwer Academic Publishers, 1987. DOI: 10. 1007/978-94-009-3687-4

[24] Priest, G., "Motivations for paraconsistent logic: The slippery slope from classical logic to dialetheism", pages 223-232 in G. Priest and D. Batens (eds.), Frontiers of Paraconsisten Logic, Research Studies Press, Baldock, 2000.

[25] Priest, G., "Vasil'iev and imaginary logic", History and Philosophy of Logic 21, 2 (2000): 135-146. DOI: 10.1080/01445340050064031

[26] Pynko, P. A., "Extensions of Hałkowska-Zając three-valued paraconsistent logic", Archive of Mathematical Logic 41, 3 (2002): 299-307. DOI: 10. $1007 / \mathrm{s} 001530100115$

[27] Restall, G., "Relevant and substructural logics", pages 289-398 in D. M. Gabbay and J. Woods (eds.), Handbook of the History and Philosophy of Logic, volume 7, Elsevier, 2006. DOI: 10.1016/S1874$5857(06) 80030-0$ 
[28] Sette, A.M., and E.H. Alves, "On the equivalence between two systems of paraconsistent logic", Bulletin of the Section of Logic 24, 3 (1995): 155157.

[29] Sette, A. M., and E. H. Alves, "On the equivalence between some systems of non-classical logic", Bulletin of the Section of Logic 25, 2 (1996): 68-72.

[30] Skura, T., "A complete syntactical characterisation of the intuitionistic logic", Reports on Mathematical Logic 23 (1989): 75-80.

[31] Skura, T., "A new criterion of decidability for intermediate logics", Bulletin of the Section of Logic 19, 1 (1990): 10-14.

[32] Skura, T., "Syntactic refutations against finite models in modal logic", Notre Dame Journal of Formal Logic 35, 4 (1994): 595-605. DOI: 10. $1305 /$ ndjfl/1040408615

[33] Skura, T., Aspects of Refutation Procedures in the Intuitionistic Logics and Related Modal Systems, Wydawnictwo Uniwersytetu Wrocławskiego, Wrocław, 1999.

[34] Skura, T., "Maximality and refutability", Notre Dame Journal of Formal Logic 45, 2 (2004): 65-72. DOI: $10.1305 /$ ndjfl/1095386644

[35] Skura, T., "Refutation systems in propositional logic", pages 115-157 in D. M. Gabbay and F. Guenthner (eds.),Handbook of Philosophical Logic, volume 16, Springer, 2011. DOI: 10.1007/978-94-007-0479-4_2

[36] Skura, T., Refutation Methods in Modal Propositional Logic, Semper, 2013.

[37] Skura, T., and R. Tuziak, "Three-valued maximal paraconsistent logics", Acta Universitatis Wratislaviensis. Logika 23 (2005): 129-134.

[38] Suchon, W., "Vasil'iev. What did he exactly do?", Logic and Logical Philosophy 7 (1999): 131-141. DOI: 10.12775/LLP.1999.011

[39] Tanaka, K., "Three schools of paraconsistency", Australasian Journal of Logic 1 (2003): 28-42.

[40] Wajsberg, M., "Untersuchungen über den Aussagenkalkül von A. Heyting", Wiadomości Matematyczne 46 (1938): 429-435.

[41] Wybraniec-Skardowska, U., "On the notion of function of the rejection of propositions", Acta Universitatis Wratislaviensis. Logika 23 (2005): 179202.

ADAM TRYBUS

Institute of Philosophy

The University of Zielona Góra, Poland

adam.trybus@gmail.com 